\title{
Methods for grafting Arabidopsis thaliana and Eutrema salsugineum
}

\author{
Yan Li๋ ${ }^{\dagger}$ Wei Sun ${ }^{\dagger}$, Fulin Liu, Jin Cheng, Xiaojie Zhang, Hui Zhang and Yanxiu Zhao*
}

\begin{abstract}
Background: Grafting, an ancient agronomic technique, is an artificial mode of asexual reproduction in plants. Recently, grafting research has gradually shifted from modifying agronomic traits to the study of molecular mechanism. Grafting is an excellent tool to study long-range signaling processes in plants. And the grafting between species will help elucidate the molecular mechanisms underlying contrasting differences between different species. Arabidopsis thaliana is a salt-sensitive model glycophyte and Eutrema salsugineum (previously Thellungiella salsuginea, salt cress) is a salt-tolerant model halophyte. Successful grafting of these two model plants will help further study the physiological and molecular mechanisms underlying salt tolerance in plants. The aim of this study was to demonstrate two sterile micro-grafting methods for Arabidopsis and salt cress.

Results: We developed the methods for sterile grafting between A. thaliana and E. salsugineum; this is the first report on inter-generic grafting between Arabidopsis and Eutrema. The method involves cut-in grafting under sterile conditions. The grafted plant part was placed in half strength Murashige and Skoog medium with 1\% agar and 1\% sugar, and then cultured vertically with $22^{\circ} \mathrm{C} / 18^{\circ} \mathrm{C}$ short-day/night cycles. The plants were then transferred to half strength Hoagland nutrient solution for hydroponics. The reported method is simple and easy to operate. Self-grafted Arabidopsis-Arabidopsis and Eutrema-Eutrema plants were used as controls, which were obtained with an improved hypocotyl-cutting grafting method. Ion contents in grafted plants were detected by inductively coupled plasma optical emission spectroscopy. The results showed that the ion content in salt cress and Arabidopsis changed to different degrees after grafting.

Conclusions: The inter-species grafting technique described here makes it possible to study hybrid plants between Arabidopsis and Eutrema and will contribute to further understanding of long-distance communications in plants. This technique also provides a reference for improving plant varieties using grafting, such as gardening plants, as well as fruit and vegetable crops.
\end{abstract}

Keywords: Arabidopsis thaliana, Cut-in grafting, Eutrema salsugineum (Thellungiella salsuginea), Sterile, ICP-OES

\section{Background}

Grafting, an old agronomic technique, is a type of vegetative/asexual propagation of plants. It is usually carried out by exploiting callus growth after plant injury. Grafting is still a common technique in the field of gardening. Grafting has been used in agriculture since $>2$ 000 years BCE (Before the Common Era) $[1,2]$ in China.

\footnotetext{
*Correspondence: zhaoyx@sdnu.edu.cn

†Yan Li and Wei Sun contributed equally to this work

Shandong Provincial Key Laboratory of Plant Stress Research, College

of Life Science, Shandong Normal University, Jinan 250014, Shandong, China
}

It is widely used in agriculture, and the cultivation of forest trees, and horticultural plants. For instance, cultivars with pathogen resistance or stress tolerance are usually used as rootstocks to improve the growth vigor of aboveground cultivars with high economic values. Grafting has also been used to break the juvenile stage in order to promote flowering in woody species. Compared to genetically modified crops, grafted plants are more palatable to the public [3]. Grafting is also a useful technique in plant scientific research. Considerable efforts in this direction started in the $70 \mathrm{~s}$ and $80 \mathrm{~s}$ of the last century. However, with the rapid development of biotechnology, the focus of grafting research has gradually shifted from improving

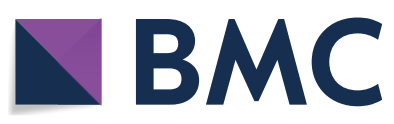

(c) The Author(s) 2019. This article is distributed under the terms of the Creative Commons Attribution 4.0 International License (http://creativecommons.org/licenses/by/4.0/), which permits unrestricted use, distribution, and reproduction in any medium, provided you give appropriate credit to the original author(s) and the source, provide a link to the Creative Commons license, and indicate if changes were made. The Creative Commons Public Domain Dedication waiver (http://creativecommons.org/ publicdomain/zero/1.0/) applies to the data made available in this article, unless otherwise stated. 
agronomic traits to the study of molecular mechanism. Studies have demonstrated that grafting is an excellent tool in elucidating mechanisms involved in long-range signaling processes within plants, such as those involved in flowering [4-7], auxin regulation [8], heavy metal tolerance [9], small RNA movement [10,11], nutrition state [10], and nuclear silencing [12, 13].

It is well known that the factors affecting graft survival rates mainly depend on the compatibility of the rootstock and scion. Compatibility refers to the similarity between the rootstock and scion with respect to structural, genetic, and physiological characteristics, and the ability to adapt to each other after grafting. The compatibility of grafts from the same plants is the strongest; the farther the relationship between plants, the weaker the compatibility between them. It is difficult to graft plants from different genera. Besides the compatibility of scions and rootstocks, the vigor of rootstock and scion is also an important factor affecting graft survival. The survival rate of grafted plants also varies with species, and the survival rate of the grafted body is different even in the same species at different ages. Furthermore, environmental factors such as temperature, humidity, and light intensity can also affect the survival rate of grafted plants. In conclusion, there are many factors that influence graft survival rate.

Arabidopsis thaliana and Eutrema salsugineum (Thellungiella salsuginea) belong to the same family Cruciferae but different genera. Arabidopsis thaliana is used as a model plant in genetic studies of dicotyledonous plants. Eutrema Salsugineum (aka. Salt cress) is an excellent salttolerant plant, and the genome of E. salsugineum has been completed [14]. Approximately $95 \%$ of the genes in E. salsugineum are similar to those expressed in Arabidopsis [15], and most of the amino acid sequences are the same as those of Arabidopsis [14]. Eutrema salsugineum has a relatively small nuclear genome, approximately twotimes of that of $A$. thaliana [16]. All the ecotypes of $E$. salsugineum show resistance to a range of environmental stresses such as cold, drought and oxidative stresses [17]. E. salsugineum has gained attention owing to its extreme salt-tolerance. Therefore, salt cress was suggested as a model halophyte for studying salt tolerance more than 20 years ago [15, 18-20]. This has opened a new window for research covering a wide range of aspects, including not only abiotic stress [21], but also photosynthesis [22], stress-related protein function [23], and surface wax production [24] using E. salsugineum as a model plant. In Arabidopsis, several grafting approaches have been developed to investigate systemic signaling [9, 25-31]. However, to the best of our knowledge, there is no report on grafting methods for E. salsugineum, or on methods for grafting between Arabidopsis and Eutrema. The difficulty of grafting between these plants is significantly high. Successful grafting of these two model plants will help provide crucial insights into molecular mechanisms underlying plant stress tolerance. Here we demonstrate two sterile micro-grafting methods for inter-generic grafting between Arabidopsis and Eutrema, with the advantages of simplicity and high survival rate.

\section{Grafting methods}

In the present study, we focus on two efficient sterile seedling micro-grafting protocols between Arabidopsis, a salt-sensitive plant, and salt cress, a salt-tolerant plant. One method was hypocotyl-hypocotyl flat-surface cutting of the seedlings, as described by Seung [26] and Turnbull et al. [25] with some modifications. The other method was cut-in grafting, which we will demonstrate herein. The abbreviation $\mathrm{A} / \mathrm{E}$ used herein indicates that $A$. thaliana scions were grafted onto $E$. salsugineum stocks, and conversely E/A indicates that $E$. salsugineum scions were grafted onto $A$. thaliana stocks. In a control experiment, self-grafting of $A$. thaliana and $E$. salsugineum was performed. The self-grafted seedlings of $A$. thaliana are referred to as A/A and the self-grafted seedlings of E. salsugineum are referred to as E/E. Optimized grafting methods were applied to different rootstocks and scions. The method of cut-in grafting was used for A/E (Fig. 1a) and the other method, i.e., hypocotylhypocotyl flat-surface cutting was used for A/A, E/E, and

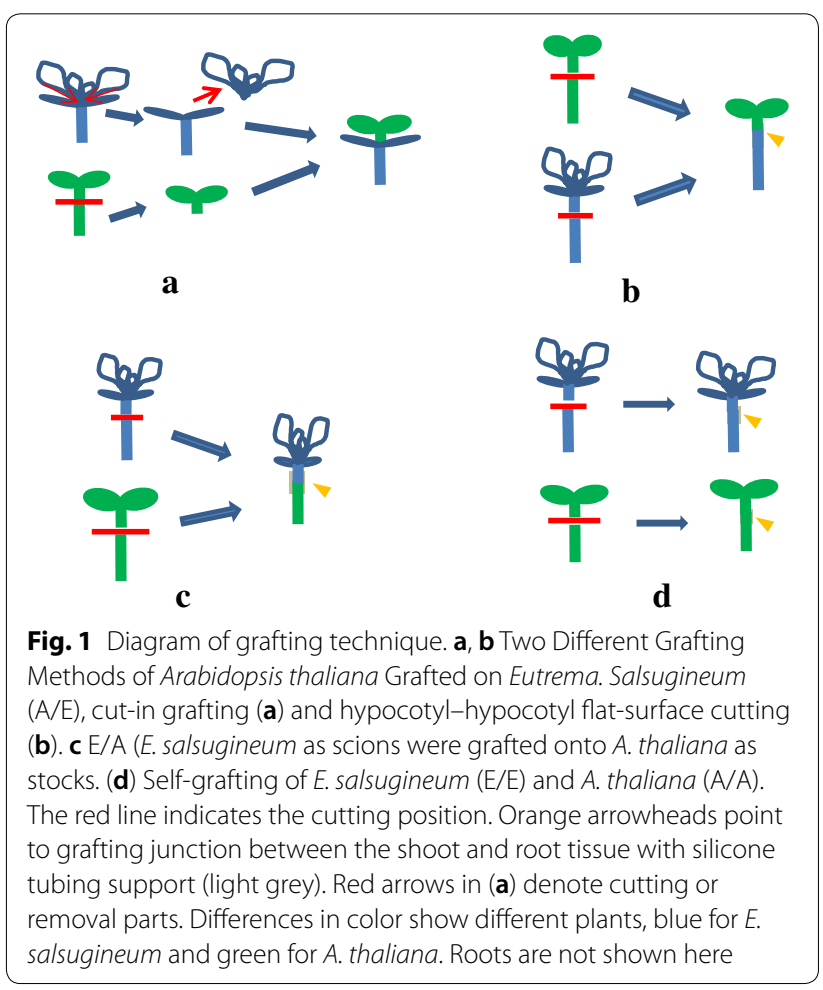


E/A (Fig. 1c, d). It is relatively easy to achieve successful graft using the conventional grafting method (i.e., hypocotyl-hypocotyl flat-surface cutting), and the subsequent growth of grafted plants is usually satisfactory. Therefore, we will not discuss this method herein.

\section{Materials and growth conditions}

Arabidopsis thaliana Columbia-0 (Col) seeds and E. salsugineum (Shandong, China) seeds used in this study are preserved and propagated in our laboratory. The plants were grown under long-day (LD 16/8 h light/dark) or short-day (SD 8/16 h light/dark) light conditions, with $22{ }^{\circ} \mathrm{C} / 18{ }^{\circ} \mathrm{C}$ day/night cycles and $100 \mu \mathrm{mol} \mathrm{m}^{-2} \mathrm{~s}^{-1}$ light intensity.

\section{Grafting procedure}

The step-by-step protocol of cut-in grafting method applied for A/E was as follows:

Preparation of rootstock and scion materials: salt cress seeds was disinfected with $0.5 \% \mathrm{NaClO}$ (sodium hydrochlorite) for 6 min followed by 4-5 rinses in distilled water, and subsequently sowed under sterile conditions on half strength Murashige and Skoog (MS) medium containing $1.0 \%$ agar and $1 \%$ sugar. One week after low temperature $\left(4{ }^{\circ} \mathrm{C}\right)$ stratification treatment, the seedlings were transferred to SD conditions and vertically cultured with light for 10-15 days (or 7-10 days after germination) as rootstock seedlings. For the germination of Arabidopsis seeds, the sterilization method and growth conditions were the same as those of E. salsugineum, except the low temperature $\left(4{ }^{\circ} \mathrm{C}\right)$ stratification treatment, which was for 2-3 days. The optimum age for grafting Arabidopsis seedlings is $3-5$ days. Because the germination time and growth rate of the two species are different, the planting times were adjusted accordingly. Usually Arabidopsis should be planted after the germination of salt cress seeds.

1. Grafting under sterile conditions (Fig. 1a): The grafting procedure was performed under anatomical microscopes in a sterile hood. E. salsugineum seedlings of uniform size were moved using tweezers to new $1.0 \%$ half strength MS agar medium. To be used as rootstocks, the true leaves and meristems were removed from salt cress seedlings with a stainless steel double-edged blade razor, while the cotyledons were retained. Under the same sterile conditions, the hypocotyl of $A$. thaliana seedings was cut transversely at approximately one-fourth of the height from the top (typically 2-3 $\mathrm{mm}$ ) and the upper part of the seedling was used as a scion. Cutting of $A$. thaliana as scions can be carried out directly in the original medium plates or in the plates where the salt cress has been prepared as rootstocks. The A. thaliana scion was grafted onto salt cress without any fixtures because the tiny amount of liquid at the cutting surfaces of the two parts is sufficient to hold them together. Afterwards, the Petri dish was swiftly but carefully sealed with parafilm to preserve humidity. Please take caution to make sure the leaves are naturally extended to prevent possible dislodging due to plant growth.

2. Culturing of grafted body: The grafted A/E complex was placed under SD conditions in a Percival growth cabinet for 6-8 days.

3. Transplantation of grafted seedlings (Fig. 2): Six to eight days after the grafting, the interface of butt joint was examined under an anatomic microscope. The successfully graft should have solid connection between the scion and the stock by this time. The seedlings were then transferred to a hydroponic system or nutritious soil per requirement. After the transplanting, attention should be paid to cut off adventitious roots in time, if the adventitious roots occur.

\section{Seed surface disinfection}

In the cut-in grafting method, the surface of seeds was disinfected directly using $0.5 \%$ sodium hydrochloride $(\mathrm{NaClO})$ for 6-8 min, and then washed with sterile water for 4-6 times. This method avoids the use of alcohol for sterilization. Moreover, the sterilization method with $\mathrm{NaClO}$ is simple, thereby reducing secondary pollution, and the seeds can germinate normally. It also avoids nongermination of seeds because of over sterilization, which affects seed germination and subsequent analyses. As long as the concentration of $\mathrm{NaClO}$ is controlled properly, over-sterilization will not occur even if the sterilization lasts for several minutes.

\section{Seedling age}

In the cut-in grafting method, slightly older E. salsugineum seedlings (7-10 days after sprouting) with lateral roots, can better meet the fast growth characteristics of their scions (A. thaliana), this also reduces the formation of adventitious roots, greatly improves the survival rate of grafts and ensure the vigorous growth of grafted seedlings at the later stage (Table 1). We found that 3-5-dayold seedlings of Arabidopsis were favorable to improve the survival rate of grafted seedlings; it also supports the vigorous growth of grafted seedlings (Table 1 ). This may be because the life cycle of $A$. thaliana is short, compared to that of E. salsugineum. Therefore the use of older $A$. thaliana seedlings as scions resulted in low survival rates; 


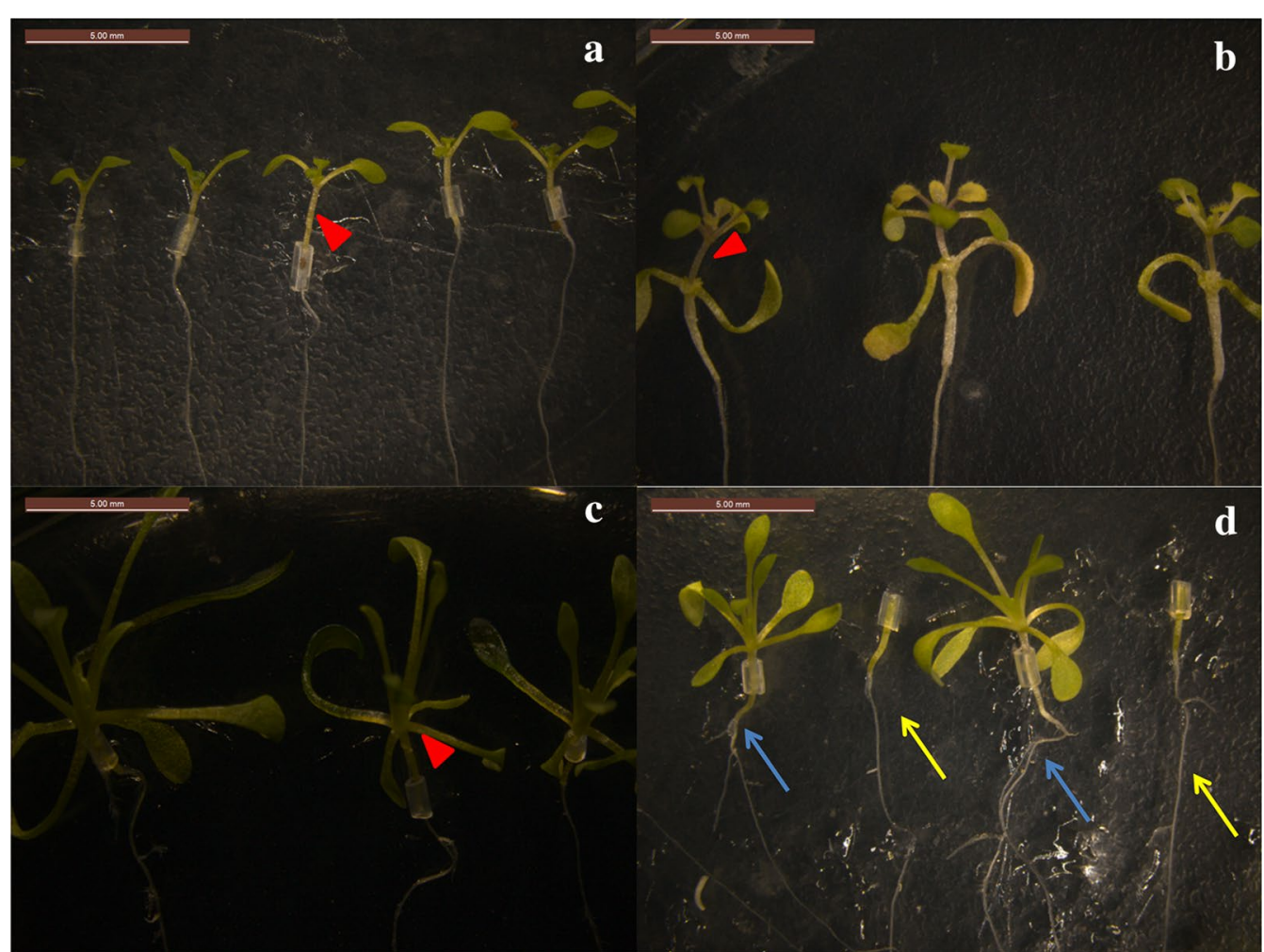

Fig. 2 Succeed grafted seedlings. a Self-grafting of A. thaliana (A/A). b A/E grafted seedlings. c Self-grafting of E. salsugineum (E/E). d E/A grafted seedlings. Red arrows in a-c denote grafting sites. Arabidopsis thaliana roots as rootstocks are shown by arrows of different colors in $\mathbf{d}$, blue arrows indicate the roots after grafting, and yellow arrows indicate the roots before grafting

Table 1 Varying the age of the seedlings on A/E grafting

\begin{tabular}{llllll}
\hline Scion age & Rootstock age & Total grafts & Grafting success & Success rate (\%) & Later growth \\
\hline $3-5$ & $3-5$ & 100 & 50 & 50 & Grow weak \\
& $7-10$ & 100 & 96 & 96 & Grow strong \\
& $11-15$ & 100 & 80 & 80 & Grow strong \\
$6-8$ & $3-5$ & 100 & 20 & 20 & Grow weak \\
& $7-10$ & 100 & 70 & 70 & General growth \\
& $11-15$ & 100 & 60 & 60 & General growth \\
\hline
\end{tabular}

even if the A/E grafts survived, the growth at later stages was poor and early flowering is usually observed.

\section{Flat-surface cutting method}

Multiple types of grafting methods have been reported for A. thaliana [26, 27, 30, 31]. In the present study, the method of cut-in grafting was only used for $\mathrm{A} / \mathrm{E}$ grafts and the method of hypocotyl-hypocotyl flat-surface cutting was used for A/A, E/E, and E/A (Fig. 1c, d). The two methods require the same culturing conditions except for the cutting site. Seedlings aged 3-5 days were used for A/A self-grafting. For E/A grafting, 7 to 10-day-old seedlings of $A$. thaliana as rootstocks are more beneficial for the survival of grafts. Similarly, 7-10 day old Seedlings were used for E/E self-grafting. We found that both the survival rate and later growth of A/E seedlings were significantly different between the two grafting methods (Fig. 1a, b and Table 2). The cut-in method is more satisfactory for $\mathrm{A} / \mathrm{E}$ grafting. The main reason might be the fast growth rate of $A$. thaliana is better accommodated using this method. When traditional flat-surface cutting was used for $\mathrm{A} / \mathrm{E}$ grafting, adventitious roots from the scion appear before the callus formation at the junction, affecting the success rate of grafted plants, because 
Table 2 Effects of two grafting methods on A/E grafting

\begin{tabular}{lllll}
\hline Grafting method & Total grafts & Grafting success & Success rate (\%) & Later growth \\
\hline $\begin{array}{l}\text { Hypocotyl-hypocotyl flat-surface } \\
\text { cutting }\end{array}$ & 80 & 10 & 12.5 & Grow weak \\
\begin{tabular}{l} 
Cut-in grafting \\
\hline
\end{tabular} & 80 & 68 & 85 & Grow strong \\
\hline
\end{tabular}

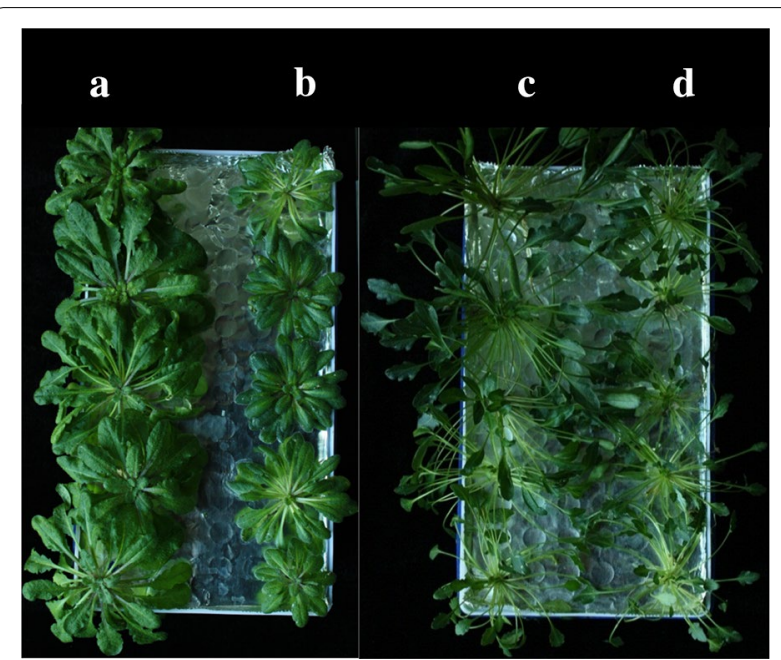

Fig. 3 Two-month grafted seedlings $(\mathbf{a}, \mathbf{b})$ different rootstocks with the same scion (Arabidopsis), as do (c, $\mathbf{d})$ except that the scion is Salt Cress. a Rootstock is Arabidopsis thaliana(A/A), b Rootstock is E. salsugineum (A/E), c Rootstock is Arabidopsis thaliana (E/A), d Rootstock is E. salsugineum (E/E)

the growth of salt cress is slower than that of A. thaliana. In comparison the success rate of the cut-in method is almost $100 \%$ as long as the cut is smooth and two parts tightly placed.

\section{Results}

\section{Successfully grafted seedlings}

After successful grafting of scions and rootstocks, it was apparent that the above-ground and under-ground parts promote the growth of each other, whereas the un-grafted rootstocks and scions remain basically unchanged (Fig. 2d).

\section{Characterization of growth}

The size, shape, and leaf pigmentation pattern of salt cress and Arabidopsis were markedly affected by grafting. The results showed that the growth of the aerial part of the A/E plants was slightly depressed; the longer the vegetative growth stage was, the more obvious the difference was in the plant sizes. Grafting resulted in smaller $A$. thaliana parts in A/E grafts compared to the A/A plants (Fig. 3a). In contrast, larger $E$. salsugineum shoots (E/A) were observed when compared to the E/E plants (Fig. 3b). Furthermore, the leaf pigmentation pattern of the scion in $\mathrm{A} / \mathrm{E}$ grafts was similar to that of the E/E homografts. Similarly the leaf color of the E. salsugineum part was similar to that of the A/A grafts. These interesting phenomena revealed that some traits associated with morphological characteristics, growth, and development characteristics were changed by grafting. Interestingly, we found that the rootstock has a profound effect on the growth and developmental characteristics of the scion. It has been suggested that grafting can be used to study the transduction of plant signals and interaction between above- and below-ground parts. Considering the high salt tolerance of E. salsugineum, it is worth studying the performance of $\mathrm{A} / \mathrm{E}$ and $\mathrm{E} / \mathrm{A}$ grafts under saline condition in the future. We observed no significant difference between the plants during the first 2 weeks after being transferred to a hydroponic system, but the difference in growth was more apparent over time. After 2 months of culture in the hydroponic system, Arabidopsis was significantly smaller than the corresponding self-grafted seedlings, when $A$. thaliana as scions were grafted onto $E$. salsugineum rootstocks. Furthermore, the size of all the rosette leaves was smaller than that of the self-grafted seedlings (Fig. 3a) and the size of epidermal cells in mature leaves also smaller (Fig. 4a). The results were opposite when compared E/A grafts to the E/E plants (Figs. 3b, $4 \mathrm{~b}$ ).

\section{Ion content determination}

We employed ICP-OES (inductively coupled plasma atomic emission spectroscopy) to determine the levels of six elements, viz., $\mathrm{Ca}, \mathrm{Fe}, \mathrm{K}, \mathrm{Mg}, \mathrm{Na}$, and $\mathrm{Zn}$, in samples obtained 2 months after grafting. ICP-OES is one of the most precise multi-element analysis technique for trace and minor metals [32] and has the advantage of measuring both macronutrients and micronutrients with a wide range of concentrations in plants. The concentration of minerals in grafted samples (Table 3 ) is expressed in micrograms per gram. The ion contents in $A$. thaliana and $E$. salsugineum clearly changed to varying degrees after they were grafted together. Our original intention of the study was to determine how salt tolerance changes when a halophyte is grafted with a glycophyte. Therefore, 


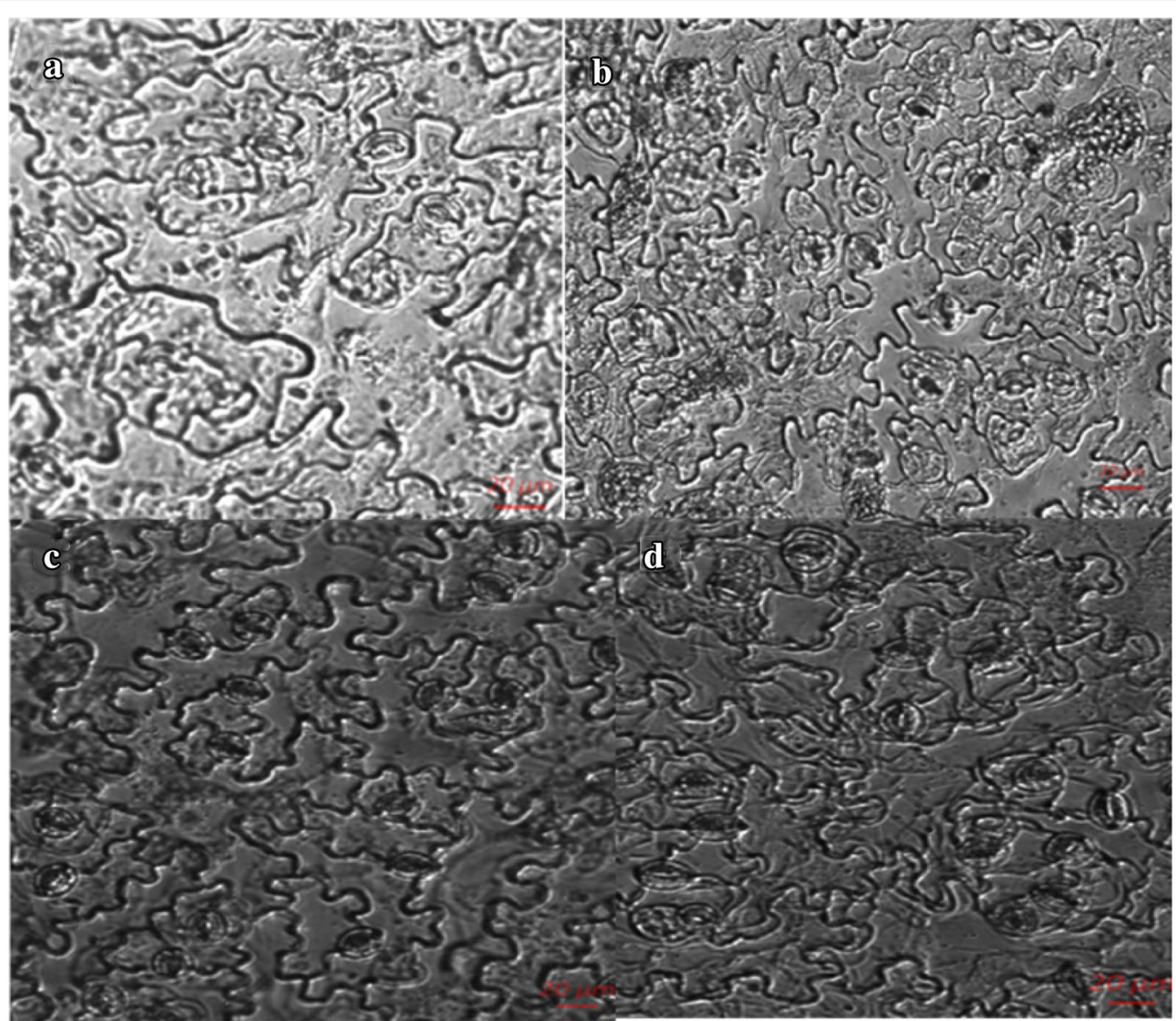

Fig. 4 Epidermal cells in mature leaves (a) Self-grafting of A. thaliana (A/A), (b) Grafted seedlings of A/E, (c) Self-grafting of E. salsugineum (E/E), (d) E/A grafted seedlings

Table 3 The concentration of minerals in grafted samples

\begin{tabular}{|c|c|c|c|c|c|c|}
\hline \multirow[t]{2}{*}{ Grafts } & \multicolumn{6}{|l|}{ Ion } \\
\hline & Potassium & Sodium & Calcium & Magnesium & Iron & Zinc \\
\hline A/A-shoot & $12,858.32 \pm 931.80$ & $1204.88 \pm 80.59$ & $36,524.40 \pm 874.03$ & $7420.49 \pm 435.10$ & $141.77 \pm 14.42$ & $38.94 \pm 3.21$ \\
\hline A/E-shoot & $10,106.16 \pm 1205.97^{d * *}$ & $1397.9 \pm 247.91$ & $39,942.75 \pm 202.28$ & $6138.81 \pm 461.11$ & $135.00 \pm 20.06$ & $35.38 \pm 1.70$ \\
\hline E/A-shoot & $14,868.31 \pm 695.20$ & $707.89 \pm 24.66$ & $37,169.66 \pm 1662.76$ & $4276.28 \pm 546.09$ & $118.95 \pm 34.96$ & $25.63 \pm 1.49$ \\
\hline E/E-shoot & $12,406.83 \pm 749.57^{a *}$ & $893.8 \pm 95.48$ & $27,259.93 \pm 1500.16^{\mathrm{a} *}$ & $3777.52 \pm 421.56$ & $121.62 \pm 20.87$ & $41.69 \pm 3.55^{\mathrm{a} * *}$ \\
\hline A/A-root & $10,171.13 \pm 528.54^{b *}$ & $1396.41 \pm 60.72^{b_{*}}$ & $2818.28 \pm 168.40^{b *}$ & $833.00 \pm 20.42^{b * *}$ & $300.34 \pm 12.82^{b *}$ & $12.42 \pm 2.07^{b * *}$ \\
\hline E/A-root & $7569.79 \pm 531.27$ & $1709.06 \pm 133.45$ & $3120.19 \pm 964.76$ & $618.87 \pm 61.50$ & $375.73 \pm 22.73$ & $32.06 \pm 3.22$ \\
\hline A/E-root & $7748.17 \pm 409.96$ & $1528.33 \pm 160.85$ & $3328.57 \pm 497.91$ & $1700.96 \pm 116.09$ & $197.54 \pm 27.08$ & $21.87 \pm 3.73$ \\
\hline E/E-root & $4163.79 \pm 210.98^{\mathrm{C**}}$ & $861.3 \pm 46.00^{\text {c***}}$ & $2617.92 \pm 458.93^{c *}$ & $1523.01 \pm 160.50$ & $196.75 \pm 10.20$ & $43.02 \pm 6.34^{c *}$ \\
\hline
\end{tabular}

Results expressed as mean \pm standard deviation $(n=3)$

${ }^{*} \mathrm{P}<0.05$; ${ }^{*} \mathrm{P}<0.01$

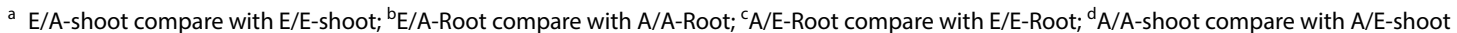

we focused on changes in sodium and potassium ion concentrations. The ratio of potassium to sodium changed to some extent after grafting.

\section{Discussion}

In addition to grafting methods and seedling age, smoothness of cutting surface, cutting site and length 
of illumination will affect the success of grafting.

\section{Cutting blade and supports}

The smoothness of the cutting surface is one of the key factors that determine the success rate of grafting. Different blades including Swann medical surgical blades \#11 and \#22 (Swann-Morton), ordinary single edge razor blade (Shanghai Gillette), and stainless steel double-edged blade razor (Shanghai Gillette) were tested in this study. We found that double-edged blade razor worked well. Its knife edge is sharp and thin, and it is good for a smooth cutting surface, causing minimal damage to the meristem and thus reducing the formation of adventitious roots and improving the survival rate of grafted seedlings. With this approach, the injury to the cut surface is minimized, which help with the quick recovery of rootstock and scion after butt-jointing. This can be attributed to the fact that, as indicated by Turnbull [25], the rapid formation of graft union diminished adventitious rooting. The double-edged blade can be re-used more than 100 times. In order to make the blade last longer and reduce the trouble of replacement, we sterilized it by soaking it in $70 \%$ alcohol instead of flaming it. However, it is important to replace the blade when it loses sharpness. We fixed the blade to a blade holder (Fig. 5), which is more conducive for cutting and for reducing contamination.

Neither link support nor cutting support was employed in this study. The scion and stock were placed together on the medium without any supporting elements. The concentration of the agar, i.e., 1.0\%, was sufficient to harden the medium to serve as the support for cutting the material. After cutting on the medium with $1.0 \%$ agar, no collar supports were used between scion and stock, allowing the healing happen in vertically oriented plates. This significantly simplifies the grafting process and shortens the time. With some practice, as many as over 30 heterografts or more than 50 homografts per hour can be achieved. This simplified method also reduces contamination rate often seen with complicated operation steps and ensure the success rate of grafting.

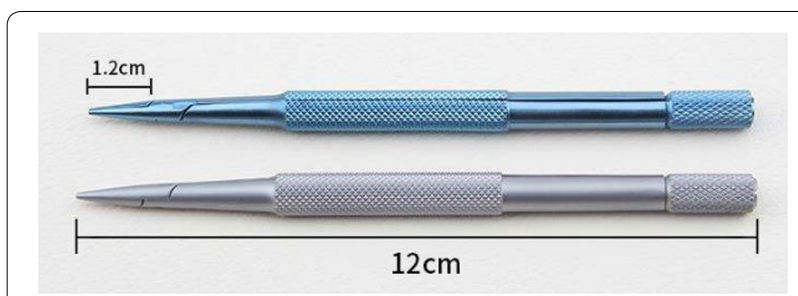

Fig. 5 Blade holder

\section{Cutting position}

It is noteworthy that the cutting position also affects the success rate of grafting. The survival rate of grafted plants was higher when the cutting site was in the upper half of the hypocotyl. We generally cut at one-fourth of length from the top of the A. thaliana hypocotyl as scion, and then grafted it onto the prepared E. salsugineum rootstock. Adventitious roots could appear above the graft junction and should be removed.

\section{Plate orientation and day light length}

Unlike other methods, we did not use support at the junction between the scion and the rootstock. They were placed together on the surface of the medium and allowed to heal in vertically oriented position. The surface of excised tissues should be in tight contact. It is easy to distinguish scions from stocks, because the grafting sites are clearly visible (Figs. 2, 6).

Quick operation is also crucial In order to keep the incision fresh. Typically 8-10 grafts were prepared on each petri dish, and then sealed with Parafilm to maintain high humidity but without contamination. It is necessary to handle the petri dishes with grafts gently so that scions from rootstocks were not dislodged.

We used SD light conditions because the hypocotyls of $A$. thaliana and E. salsugineum elongate faster compared to under LD light condition. Long hypocotyls are not only useful for cutting, but also convenient for grafting on a plate. Furthermore, SD light cycle is necessary for the growth of seedlings after grafting. It is well known that SD conditions promote the vegetative phase of $A$. thaliana, which help connect the calluses at the junction and avoid adventitious root growth.

After the grafting the plants were grown and allowed to recover from the grafting procedure under SD conditions in a Percival growth cabinet. After confirming that the grafting is successful under this condition (approximately 8-10 days after the grafting procedure), the grafts were transferred to a hydroponic system, and further experiments were also performed under SD light condition, in order to maintain the growth rate of grafted plants relatively consistent in later stages.

Seeds can be germinated under LD or SD light conditions, but after grafting the seedlings should be cultured under SD. In the cut-in grafting method, SD light condition can not only improve the survival rate of grafted plants, but also benefit the growth of grafted seedlings at later stages of development. This is because the growth cycle of $A$. thaliana is shorter compared to E. salsugineum. After the cut (i.e. injury), the flowering time of A. thaliana will be further promoted. Indeed LD light conditions lead to small plants and early flowering of the 


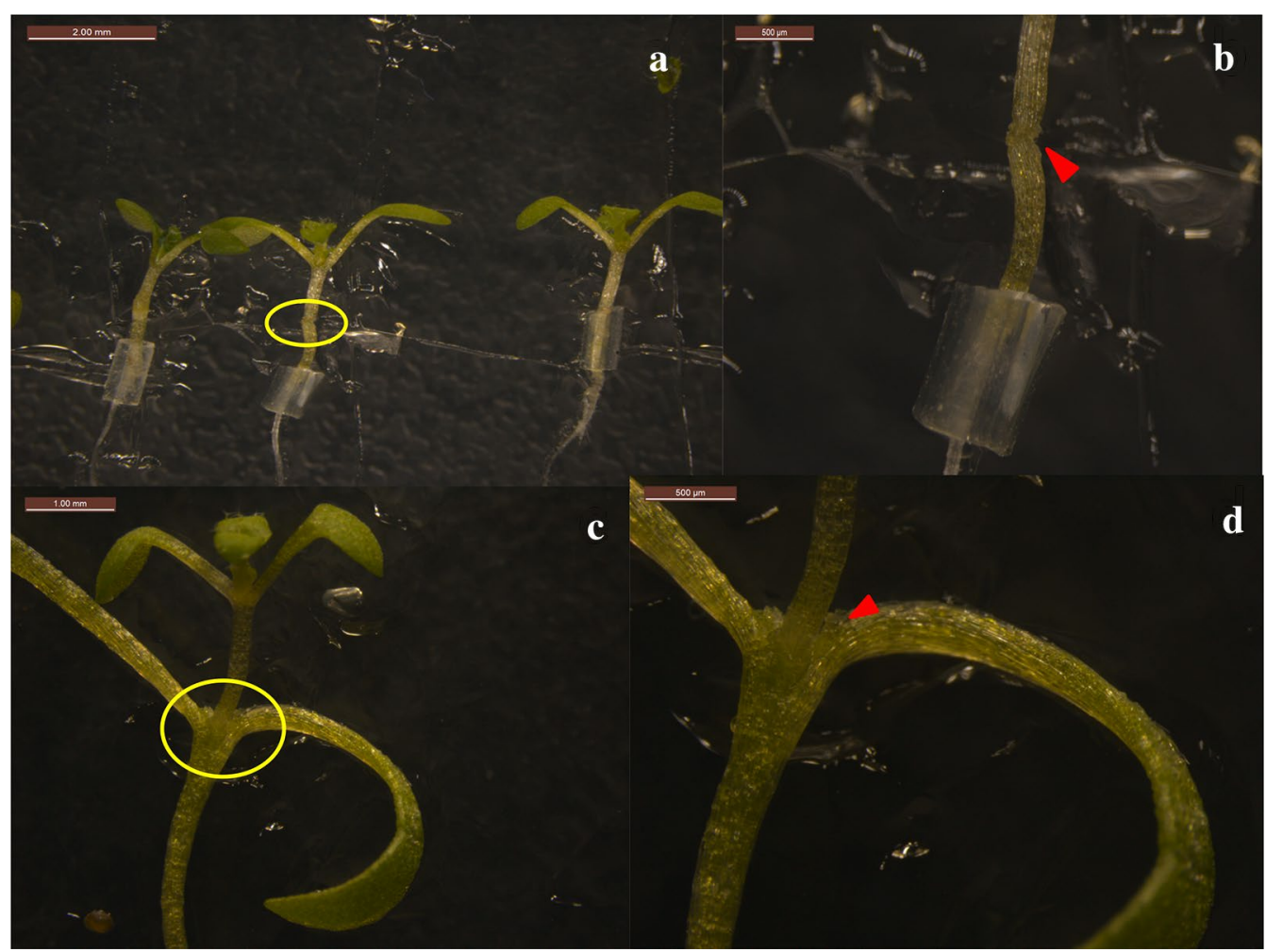

Fig. 6 The grafting sites (a) succeed grafted seedlings of A/A. b Enlargement of yellow ellipse label in (a). b succeed grafted seedlings of A/E. d Enlargement of yellow ellipse label in c

grafted seedlings, and early completion of the life cycle. SD light conditions prolong the vegetative phase and give the grafts sufficient time to recover from the injury.

\section{Conclusions}

Arabidopsis thaliana is a popular model for plant molecular biology studies, but it is a glycophyte. Although many genes involved in plant stress signaling have been identified using this system, manipulating none of them is sufficient to bring salt tolerance of $A$. thaliana to the same level as E. salsugineum, which was proposed as a model halophyte $\sim 20$ years ago. The study of physiological and molecular mechanisms underlying salt tolerance in E. salsugineum will enable better understanding of the salt tolerance mechanism of plants. The reports on grafting across different genera is limited. This is the first report on inter-generic mutual grafting between salt cress and Arabidopsis. The grafting methods described here will guarantee plant grafting for the study of shoot-root interaction mechanisms, especially the molecular mechanism underlying long range salt transport in plants.

\section{Authors' contributions}

$Y L$ and WS did major experimental work and was involved together with YXZ and $\mathrm{HZ}$ in experimental design and protocol optimization. JC, FL and XZ contributed with experimental work at the validation stage of the optimized protocol. YL, WS and YXZ drafted the manuscript, HZ edited the English language of this manuscript, which all authors read and approved. All authors read and approved the final manuscript.

\section{Funding}

This work was supported by the National Natural Science Foundation of China (31770290), Shandong Natural Science Foundation of Shandong Province (ZR2017MC035) and National College Students Innovation Project.

\section{Availability of data and materials}

The datasets used and/or analyzed during the current study are available from the corresponding author on reasonable request.

\section{Ethics approval and consent to participate} Not applicable.

\section{Consent for publication \\ Not applicable.}

\section{Competing interests}

The authors declare that they have no competing interests.

Received: 7 March 2019 Accepted: 2 August 2019

Published online: 13 August 2019

\section{Acknowledgements}

We thank the anonymous reviewers for their valuable comments and suggestions. 


\section{References}

1. Hartman $\mathrm{TH}$, Kester DE. Plant propagation-principles and practices. Prentice Hall of India Pvt Ltd: New Delhi; 1972. p. 368-9.

2. Abirami K, Mathew PA, Sasikumar B, Rema J, Baskaran V. Graft Induced genetic changes and its mechanism in bell pepper (Capsicum annuum) a review. Agric. Rev. 2009;30(4):283-92.

3. Haroldsen VM, Szczerba MW, Atkas H, Lopez-Baltazar J, Odias MJ, Chi-Ham $\mathrm{CL}$, et al. Mobility of transgenic nucleic acids and proteins within grafted rootstocks for agricultural improvement. Front Plant Sci. 2012;3:39.

4. Yoo SJ, Hong SM, Jung HS, Ahn JH. The cotyledons produce sufficient FT protein to induce flowering: evidence from cotyledon micrografting in Arabidopsis. Plant Cell Physiol. 2013;54(1):119-28.

5. Corbesier L, Vincent C, Jang S, Fornara F, Fan Q, Searle I, Giakountis A, Farrona S, Gissot L, Turnbull C, et al. FT protein movement contributes to long-distance signaling in floral induction of Arabidopsis. Science. 2007:316(5827):1030-3.

6. Ayre BG, Turgeon R. Graft transmission of a floral stimulant derived from constans. Plant Physiol. 2004;135(4):2271-8.

7. Lin MK, Belanger H, Lee YJ, Varkonyi-Gasic E, Taoka K, Miura E, Xoconostle Cazares B, Gendler K, Jorgensen RA, Phinney B, et al. FLOWERING LOCUS T protein may act as the long-distance florigenic signal in the cucurbits. Plant Cell. 2007;19(5):1488-506.

8. Bennett T, Sieberer T, Willett B, Booker J, Luschnig C, Leyser O. The Arabidopsis MAX pathway controls shoot branching by regulating auxin transport. Curr Biol. 2006;16(6):553-63.

9. Chen A, Komives EA, Schroeder Jl. An improved grafting technique for mature Arabidopsis plants demonstrates long-distance shootto-root transport of phytochelatins in Arabidopsis. Plant Physiol. 2006:141(1):108-20.

10. Buhtz A, Pieritz J, Springer F, Kehr J. Phloem small RNAs, nutrient stress responses, and systemic mobility. BMC Plant Biol. 2010;10:64.

11. Molnar A, Melnyk CW, Bassett A, Hardcastle TJ, Dunn R, Baulcombe DC. Small silencing RNAs in plants are mobile and direct epigenetic modification in recipient cells. Science. 2010;328(5980):872-5.

12. Liang D, White RG, Waterhouse PM. Gene silencing in Arabidopsis spreads from the root to the shoot, through a gating barrier, by template-dependent, nonvascular, cell-to-cell movement. Plant Physiol. 2012;159(3):984-1000

13. Brosnan CA, Mitter N, Christie M, Smith NA, Waterhouse PM, Carroll BJ. Nuclear gene silencing directs reception of long-distance mRNA silencing in Arabidopsis. Proc Natl Acad Sci USA. 2007;104(37):14741-6.

14. Oh DH, Dassanayake M, Haas JS, Kropornika A, Wright C, d'Urzo MP, Hong H, Ali S, Hernandez A, Lambert GM, Inan G, Galbraith DW, Bressan RA, Yun DJ, Zhu JK, Cheeseman JM, Bohnert HJ. Genome structures and halophyte-specific gene expression of the extremophile Thellungiella parvula in comparison with Thellungiella salsuginea (Thellungiella halophila) and Arabidopsis. Plant Physiol. 2010;154:1040-52.

15. Wang ZL, Li PH, Fredricksen M, Gong ZZ, Kimd CS, Zhang CQ, Bohnert HJ, Zhu JK, Bressan RA, Hasegawa PM, Zhao YX, Zhang H. Expressed sequence tags from Thellungiella halophila, a new model to study plant salt-tolerance. Plant Sci. 2004;166:609-16.

16. Inan G, Zhang Q, Li PH, Wang ZL, Cao ZY, Zhang H, Zhang CQ, Tanya M, Quist S, Goodwin M, Zhu JH, Shi HH, Damsz B, Charbaji T, Gong QQ, Ma
SS, Fredricksen M, Galbraith WD, Jenks AM, Rhodes D, Hasegawa MP, Bohnert HJ, Joly RJ, Bressan RA, Zhu JK. Salt cress, a Halophyte and cryophyte Arabidopsis relative model system and its applicability to molecular genetic analyses of growth and development of extremophiles. Plant Physiol. 2004;135:1718-37.

17. Wong CE, Li Y, Whitty BR, Diaz-Camino C, Akhter SR, Brandle JE, Golding GB, Weretilnyk EA, Moffatt BA, Griffith M. Expressed sequence tags from the Yukon ecotype of Thellungiella reveal that gene expression in response to cold, drought and salinity shows little overlap. Plant Mol Biol. 2005;58:561-74.

18. Bressan RA, Zhang CQ, Zhang H, Hasegawa PM, Bohnert HJ, Zhu JK. Learning from the Arabidopsis experience. The next gene search paradigm. Plant Physiol. 2001;127:1354-60.

19. Zhu JK. Plant salt tolerance. Trends Plant Sci. 2001:6:66-71.

20. Volkov V, Wang B, Doming PJ, Fricke W, Amtmann A. Thellungiella halophila a salt relative of Arabidopsis thaliana, possesses effective mechanisms to discriminate between potassium and sodium. Plant Cell Environ. 2003;27:1-14.

21. Stepien P, Johnson GN. Contrasting responses of photosynthesis to salt stress in the glycophyte Arabidopsis and the halophyte Thellungiella: role of the plastid terminal oxidase. Plant Physiol. 2009;149:1154-65.

22. Malik VM, Lobo JM, Stewart C, Irani S, Todd CD, Gray GR. Growth irradiance affects ureide accumulation and tolerance to photoinhibition in Eutrema salsugineum (Thellungiella salsuginea). Photosynthetica. 2016:54:93-100.

23. Liu XX, Fu C, Yang WW, Zhang Q, Fan H, Liu J. The involvement of TsFtsH8 in Thellungiella salsuginea tolerance to cold and high light stresses. Acta Physiol Plant. 2016;38:62.

24. Sun W, Li Y, Zhao YX, Zhang H. The TsnsLTP4, a nonspecific lipid transfer protein involved in wax deposition and stress tolerance. Plant Mol Biol Rep. 2015:33:962-74.

25. Turnbull CGN, Booker JP, Leyser HMO. Micrografting techniques for testing long-distance signalling in Arabidopsis. Plant J. 2002;32(2):255-62.

26. Rhee SY, Somerville CR. Flat-surface grafting in Arabidopsis thaliana. Plant Mol Biol Rep. 1995;13(2):118-23.

27. Nisar N, Verma S, Pogson B, Cazzonelli C. Inflorescence stem grafting made easy in Arabidopsis. Plant Methods. 2013;8(1):50.

28. Bainbridge K, Bennett T, Turnbull C, Leyser O. Grafting. Meth. Mol Biol. 2006;323:39-44.

29. Turnbull CG. Grafting as a research tool. Meth Mol Biol. 2010;655:11-26.

30. Huang Nien-Chen, Tien-Shin Yu. A pin-fasten grafting method provides a non-sterile and highly efficient method for grafting Arabidopsis at diverse developmental stages. Plant Methods. 2015;11:38.

31. Marsch-Martínez, et al. An efficient flat-surface collar-free grafting method for Arabidopsis thaliana seedlings. Plant Methods. 2013;9:14.

32. Alagić SČ, TošićSB Dimitrijević MD, Petrović JV, Medić DV. Chemometric evaluation of trace metals in Prunus persica L. Batech and Malus domestica from Minićevo (Serbia). Food Chem. 2017;2017(217):568-75.

\section{Publisher's Note}

Springer Nature remains neutral with regard to jurisdictional claims in published maps and institutional affiliations.

Ready to submit your research? Choose BMC and benefit from

- fast, convenient online submission

- thorough peer review by experienced researchers in your field

- rapid publication on acceptance

- support for research data, including large and complex data types

- gold Open Access which fosters wider collaboration and increased citations

- maximum visibility for your research: over 100M website views per year

At $\mathrm{BMC}$, research is always in progress.

Learn more biomedcentral.com/submissions 